\title{
Persistent Thrombocytosis in a Case of Pseudomyxoma Peritonea Post CRS and HIPEC -A Case Report
}

\author{
Asmaa El Hussainy*, Asit Mohanty and Jasem Al Barrak \\ Department of Medical Oncology, KCCC, Kuwait
}

Submission: November 11,2020; Published: November 19, 2020

*Corresponding author: Asmaa El Hussainy, Department of Medical Oncology, KCCC, Kuwait

\begin{abstract}
Background: Cytoreductive surgery (CRS) followed by hyper thermic intraperitoneal chemotherapy (HIPEC) is an evolving treatment for peritoneal carcinomatosis (PC). Mitomycin C (MMC), an alkylating agent, is presently the most commonly used chemotherapeutic agent for hyper thermic intraperitoneal treatment. The spleen has a role in the hematologic response after HIPEC and that splenectomy may enhance hematologic toxicity profiles of MMC. We are presenting a case who developed persistent thrombocytosis After Cytoreductive surgery followed by HIPEC.
\end{abstract}

Case presentation: A 48-year-old lady was referred to hematology OPD for first time with findings of persistent thrombocytosis on routine $\mathrm{CBC}$ follow-up. She underwent Cytoreductive surgery which include left and right parietal peritonectomy, pelvic peritonectomy, TAH+BSO, small bowel resection, right hemicolectomy, splenectomy plus heated intra operative peritoneal chemotherapy for a low-grade mucinous adenocarcinoma. Her initial platelet count was $1520 \times 10^{\wedge} 9 / \mathrm{L}$ for which she was on aspirin .Follow-up CBC showed a platelet count of $1609 \times 10^{\wedge} 9 / \mathrm{L}$. She was investigated further and there was absence of any cause of essential thrombocytosis.

Conclusion: This article to emphasize that Persistent reactive thrombocytosis can occur in a case of peritoneal carcinomatosis after CRS followed by HIPEC.

Keywords: Peritoneal carcinomatosis; Cytoreductive surgery; HIPEC

\section{Introduction}

Cytoreductive surgery (CRS) followed by hyper thermic intraperitoneal chemotherapy (HIPEC) is an evolving treatment for peritoneal carcinomatosis (PC). Mitomycin C (MMC), an alkylating agent, is presently the most commonly used chemotherapeutic agent for hyper thermic intraperitoneal treatment. This may cause a fluctuation in platelet level postoperatively, but persistent reactive thrombocytosis is rare and very few articles are available on this topic. We are presenting a case where there is persistent reactive thrombocytosis followed by CRS and HIPEC.

\section{Case Presentation}

This A 48-year-old lady, With past medical history of diabetes mellites, cholecystectomy and previous ectopic pregnancy. was referred to hematology OPD for first time with findings of persistent thrombocytosis on routine CBC follow-up. March 2013: She underwent Cytoreductive surgery which include left and right parietal peritonectomy, pelvic peritonectomy, TAH+BSO, small bowel resection, right hemicolectomy, splenectomy plus heated intra operative peritoneal chemotherapy for a low-grade mucinous adenocarcinoma. Her case was further complicated by wound infection and dehiscence that was managed by antibiotic and dressing. She remained under annual follow up with oncology center. Her initial platelet count was $1520 \times 10^{\wedge} 9 / \mathrm{L}$ for which she was on aspirin $81 \mathrm{mg}$ post splenectomy and sepsis induced reactive thrombocytosis was suspected, Follow-up CBC showed a platelet count of $1609 \times 10^{\wedge} 9 / \mathrm{L}$, She had an added thrombocytosis $18.0 \times 10^{\wedge} 9 / \mathrm{L}$. At this stage essential thrombocytosis was suspected.

She underwent Bone marrow study in June 2014 which showed no features of essential Thrombocytosis, in addition to a negative JAK2 mutation screen. In view that the patient has persistent thrombocytosis in absence of features of essential thrombocytosis in bone marrow study it was considered to be reactive and advised for further genetic screening to include CALR 
and MPL which came also negative so she was advised to continue aspirin prophylaxis. she had completed 5 years follow up for her malignancy without recurrence.

\section{Discussion}

Pseudomyxoma peritonea(PMP) arerare tumorscharacterized by large volume, ascites. These tumors are most commonly caused by epithelial neoplasm of the appendix however can also originate from mucinous tumors of the colon, rectum, or ovaries. Unfortunately, patients are often asymptomatic in the initial stage, but vague symptoms can occur [1,2]. Initial treatment of PMP is complete Cytoreductive surgery (CRS) combined with HIPEC. HIPEC consists typically of Mitomycin C however dose and timing differ between centers. Thrombocytosis can be classified into two types: essential thrombocytosis, a myeloproliferative disorder of the bone marrow, and reactive thrombocytosis, also called secondary thrombocytosis. This latter is the most common type and appears after acute inflammatory, infectious, neoplastic and stress processes. In these scenarios the levels of thrombopoietin, interleukin- 6 and catecholamines are very high, and are thought to be responsible for the increased number of platelets. Signs and symptoms of the underlying disease usually accompany reactive thrombocytosis. In lung cancer patients, reactive thrombocytosis has a prevalence as high as $30 \%$ and has been linked with tumor extension or metastatic disease, and therefore with a poor prognosis. Reactive thrombocytosis may also appear with incidences of iron deficiency (6-12\%), autoimmune disease (4$11 \%)$, cancer (1-3\%) or drug-induced problems.

The reactive thrombocytosis found in patients with systemic inflammatory diseases is not the product of the isolated action of thrombopoietin, but its interaction with other plasma cytokines such as interleukin-6 [3]. Although the diagnostic tests to differentiate essential and reactive thrombocytosis are not easy to perform, laboratory tests that show increased acute phase reactants such as C-reactive protein, fibrinogen, erythrocyte sedimentation rate and interleukin- 6 may be useful in the diagnosis of reactive thrombocytosis [4]. It is accepted that lower levels of platelets 1.000.000. $\mu \mathrm{L}-1$ are a benign condition, although it remains unclear if these findings are associated with an increased post-operative thromboembolic or hemorrhagic risk. Prophylactic treatment with platelet inhibitors in these situations is controversial, although some authors do consider management of low-dose acetylsalicylic acid [5,6].

A study by Carlos parez et al. showed that increasing the initial drug concentration in the peritoneum and/or extending the HIPEC duration leads to a greater fluctuation in platelet counts. Reactive thrombocytosis can occur post splenectomy but usually transient. Some Case reports and Studies have shown that post CRS and HIPEC, there is fluctuation in Platelet level temporarily but Persistent thrombocytosis in a case of PMP post Cytoreductive surgery and HIPEC is rare.

\section{Conclusion}

Reactive thrombocytosis can occur post splenectomy but usually transient. Many case reports and studies have shown that post CRS and HIPEC, there is fluctuation in Platelet level temporarily but Persistent thrombocytosis in a case of PMP post Cytoreductive surgery and HIPEC is rare. So we should exclude the essential thrombocytosis but at same time can keep in mind that persistent thrombocytosis can occur in cases of PMS who are undergoing CRS and HIPEC.

\section{References}

1. Mittal R, Chandramohan A, Moran B (2017) Pseudomyxoma peritonei: natural history and treatment. Int J Hyperthermia 33(5): 511-519.

2. Legue LM, Creemers GJ, de Hingh, Valery E P P Lemmens, Clément J Huysentruyt (2019) Review: pathology and its clinical relevance of mucinous Appendiceal Neoplasm and Pseudomyxoma Peritonei. clinical Coloreactal Cancer 18(1): 1-7.

3. Folman CC, Ooms M, Kuenen BB, S M de Jong, R J Vet, et al. (2001) The role of thrombopoietin in post-operative thrombocytosis. Br J Haematol 114(1): 126-133.

4. Powner DJ, Hoots WK (2008) Thrombocytosis in the NICU. Neurocrit Care 8(3): 471-475.

5. Leibovitch I, Ben Chaim J, Raviv G (1993) Quantitative charges in platelet counts following major urological pelvic surgery. Eur Urol 24: 350-354.

6. Vannucchi AM, Barbui T (2007) Thrombocytosis and thrombosis. Hematology Am Soc Hematol Educ Program 2007: 363-370.
Your next submission with Juniper Publishers will reach you the below assets

- Quality Editorial service

- Swift Peer Review

- Reprints availability

- E-prints Service

- Manuscript Podcast for convenient understanding

- Global attainment for your research

- Manuscript accessibility in different formats ( Pdf, E-pub, Full Text, Audio)

- Unceasing customer service

Track the below URL for one-step submission https://juniperpublishers.com/online-submission.php 